\title{
Single-ElectronTransistor Logic for High Reliability of Moore's Law and Low Power VLSI
}

\author{
P. Vidya Sekhar ${ }^{1}$, M. Saran Chowdary ${ }^{2}$, A.Vamsidhar ${ }^{3}$, P.V.S.Sri Ram ${ }^{4}$, N.Venkateswarulu ${ }^{5}$ \\ Student, Electronics and Communication Engineering, Sir C R Reddy College of Engineering, Eluru, India ${ }^{1,2,3,4,5}$
}

\begin{abstract}
The observation made in 1965 by Gordon Moore, co-founder of Intel, that the number of transistors that are embedded per square inch on integrated circuits had doubled every 18 months since the integrated circuit was invented. But as per latest trends in VLSI the channel width cannot be less than equal to $18 \mathrm{~nm}$, so Moore's law came to deadline in recent years. This can be avoided by using SET whose channel width $\sim 1 \mathrm{~nm}$. Recent research in SET gives new ideas which are going to revolutionise the random access memory and digital data storage technologies too. Single-electron transistor (SET) is a key element of current research area of nanotechnology which can offer low power consumption and high operating speed. The single electron transistor is a new type of switching device that uses controlled electron tunnelling to amplify current.
\end{abstract}

Keywords: VLSI, SET, Moore's Law, Island, Tunnelling effect.

\section{INTRODUCTION}

The single electron transistor or SET is type of switching device that uses controlled electron tunneling to amplify current. A SET is made from two tunnel junctions that share a common electrode. A tunnel junction consists of two pieces of metal separated by a very thin $(\sim 1 \mathrm{~nm})$ insulator. The only way for electrons in one of the metal electrodes to travel to the other electrode is to tunnel through the insulator. Since tunneling is a discrete process, the electric charge that flow through the tunnel junction flows in multiples of $\mathrm{e}(\mathrm{q}=\mathrm{ne})$, where $\mathrm{e}$ is the charge of a single electron.

\section{STructure OF SET}

The single electron transistor is made of an island connected through two tunneling junctions to a drain and a source electrode, and through a capacitor to a gate electrode (Figure 1). When there is no bias on any electrode, electrons in the system do not have enough energy to tunnel through the junctions.

A conventional field-effect transistor, the kind that makes all modern electronics Work, is a switch that turns on when electrons are added to a semiconductor and turns off when they are removed. These on and off states give the ones and zeros that digital computers need for calculation. Interestingly, these transistors are almost completely classical in their physics. Only a few numbers that characterize their behavior are affected by quantum mechanics. However, if one makes a new kind of transistor, in which the electrons are confined within a small volume and communicate with the electrical leads by tunneling, all this changes. One then has a transistor that turns on and off again every time one electron is added to it, we call it a single electron transistor (SET).

\section{WORKING OF SET}

In a single electron transistor, a drain and source electrode are connected through a tunneling junction to an island, which is also capacitively connected to a gate.
When all the biases are zero, electrons do not have enough energy to tunnel through the junction. However, if you increase the bias, but keep it less than the coulomb gap voltage, increasing the gate bias above the point of maximum slope on the coulomb staircase causes the state with one or zero excess electrons on the island to have the same energy, resulting in the coulomb barrier being removed and allowing electrons to tunnel through the junctions and between the source and the drain. The

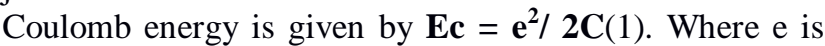
the charge on an electron and $\mathrm{C}$ is the total capacitance of the source and drain junctions and the gate capacitor. When the bias between the source and drain is greater than $\mathrm{e} / \mathrm{C}$ (e/2C across each junction), called the Coulomb gap voltage, electrons actively tunnel across the junctions, resulting in a current through the transistor independent of the gate bias.

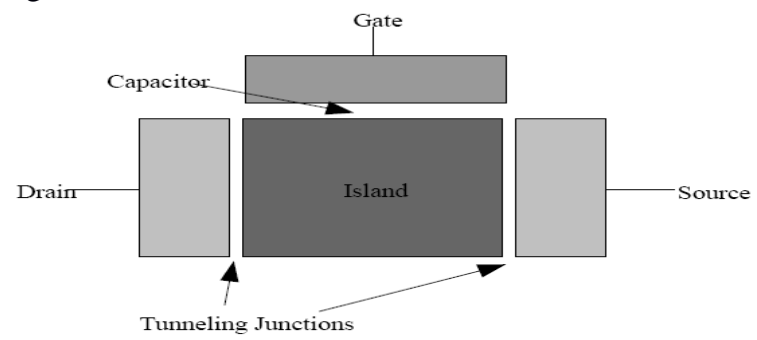

Figure1: Single-Electron transistor

In quantization of electron flow, known as the Coulomb staircase, the thermal energy of the system must be much less than the Coulomb energy. As the gate voltage increases, current increases in quantized chunks. This means that in order for a single electron transistor to operate at room temperature,

$\mathrm{kT}<<\mathrm{e}^{2} / 2 \mathrm{C}$

$\mathrm{C}<<\mathrm{e}^{2} / 2 \mathrm{kT} \approx 3.09 \times 10-18 \mathrm{~F}$

The capacitance $\mathrm{C}$ must be much less than $3.09 \times 10-18$ Farads. The capacitance is related to the distance between 
the two sides of the junction, giving that $\mathrm{C}<<3.09 \times 10-18 \mathrm{~F}$ implies $\mathrm{d}<10 \mathrm{~nm}$. The diameter of the island (quantum dot), $\mathrm{d}$, must be less than 10 nanometers

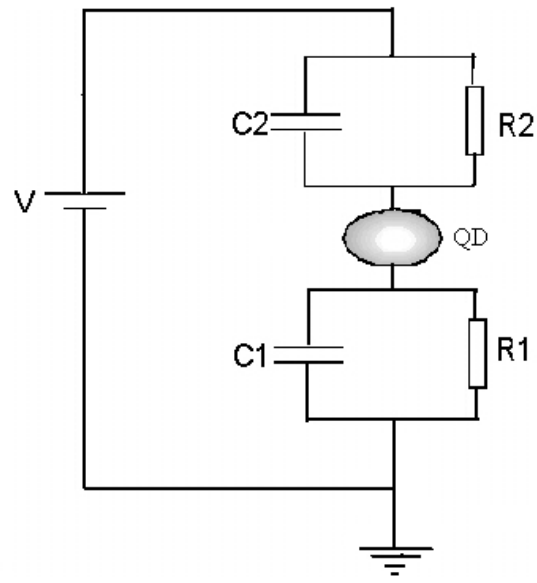

Figure 2: Island Structure

The resistance is determined by the electron tunneling and the capacitance depends on the size of the particle. We denote the resistors and capacitors by $\mathrm{R} 1, \mathrm{R} 2, \mathrm{C} 1$ and $\mathrm{C} 2$, and the applied voltage between the electrodes by $\mathrm{V}$. We will discuss how the current, I depends on V. When we start to increase $\mathrm{V}$ from zero, no current can flow between the electrodes because movement of an electron onto (charging) or off (discharging) from an initially neutral nano particle cost energy by an amount given by (1). This suppression of electron flow is called Coulomb blockade. Current start to flow through the nano particles only when the appliedvoltage $\mathrm{V}$ is large enough to establish a voltage $E$ at the nano particles such that $E>=E c$

\section{V-I ChaRACTERISTICS}

This voltage is called threshold voltage and denoted by $\mathrm{V}^{\text {th }}$. So in the $\mathrm{I}-\mathrm{V}$ curve, we expect a flat zero-current regime with a width of $2 \mathrm{~V}^{\text {th }}$. When the applied voltage reaches $\mathrm{V}^{\text {th }}$, an electron is added to (removed from) the nano particles. Further increasing the voltage, the current does not increase proportionally because it requires us to add (or remove) two electrons onto the nano particles, which cost a greater amount of energy. Once the applied voltage is large enoughto overcome the Coulomb energy of two electrons, the current starts to increase again. Thisleads to a stepwise increase in I-V curve, called Coulomb staircase (Figure 3).

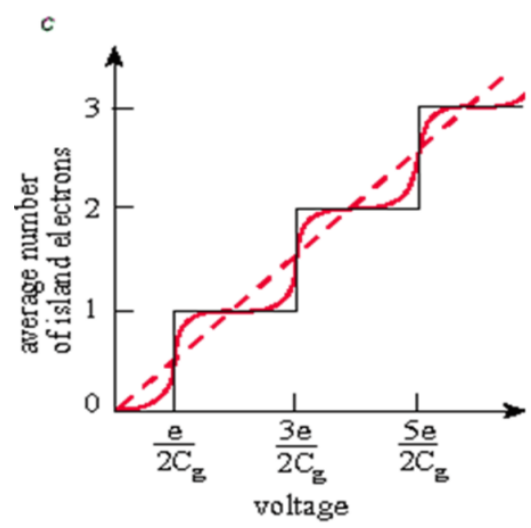

Figure3: I-V Curve

\section{Conclusions}

Single electron transistor some how extends the reliability of moore's law since the channel width is in the order of $1 \mathrm{~nm}$.

$>\quad$ In the age of nanotechnology to provide low power consumption and high operating speed in the field of VLSI design for the fabrication of various electronic devices.

$>\quad$ Resistance of SET is determined by the electron tunnelling and the capacitance depends upon the size of the nano particle.

$>\quad$ SETs could be used for memory device. The future does look bright for these devices

\section{REFERENCES}

[1]. "Single Electron Transistor Applications and Limitations" by Anil Kumar and Dharmender Dubey. Advance in Electronic and Electric Engineering ISSN 2231-1297, Volume 3, Number 1 (2013), pp. 57-62, (C) Research India Publications

[2]. "Single Electron Transistor App. \& Problems" by Om Kumar and Manjit aur. International journal of VLSI design \& Communication Systems (VLSICS) Vol.1, No.4, December 2010

[3]. M. A. Kastner, "The single electron transistor and artificial atoms", Ann. Phy. (Leipzig), vol. 9,pp. 885-895, 2000.

[4] Andreas Scholze, "Simulation of single-electron devices," Ph.D. dissertation, Univ. of Jena, Germany, 2000.

[5]. "Single Electron Transistor" by M.Eric Fombah

[6]. "VLSI Technology" by Simon Sze..

[7]. Quantum information technology based on single electron dynamics, NIT basic research laboratories Atsugi-shi, 243-0198 Japan, Vol. 1 No.3 June 2003. 